Article

\title{
FERMI: Present and Future Challenges
}

\author{
Luca Giannessi ${ }^{1,2, *}$ and Claudio Masciovecchio ${ }^{2}$ \\ 1 ENEA C.R. Frascati, Via E. Fermi 45, Frascati, 00044 Rome, Italy \\ 2 Elettra Sincrotrone Trieste, I-34149 Basovizza, Trieste, Italy; claudio.masciovecchio@elettra.eu \\ * Correspondence: luca.giannessi@elettra.eu; Tel.: +39-04-0375-8043 \\ Academic Editor: Kiyoshi Ueda
}

Received: 12 May 2017; Accepted: 12 June 2017; Published: 21 June 2017

\begin{abstract}
We present an overview of the FERMI (acronym of Free Electron laser Radiation for Multidisciplinary Investigations) seeded free electron laser (FEL) facility located at the Elettra laboratory in Trieste. FERMI is now in user operation with both the FEL lines FEL-1 and FEL-2, covering the wavelength range between $100 \mathrm{~nm}$ and $4 \mathrm{~nm}$. The seeding scheme adopted for photon pulse production makes FERMI unique worldwide and allows the extension of table top laser experiments in the extreme ultraviolet/soft X-ray region. In this paper, we discuss how advances in the performance of the FELs, with respect to coherent control and multi-colour pulse production, may push the development of original experimental strategies to study non-equilibrium behaviour of matter at the attosecond-nanometer time-length scales. This will have a tremendous impact as an experimental tool to investigate a large array of phenomena ranging from nano-dynamics in complex materials to phenomena that are at the heart of the conversion of light into other forms of energy.
\end{abstract}

Keywords: free electron laser; non-linear optics; four wave mixing; coherent control

\section{Introduction}

The newest light sources, extreme ultraviolet (XUV) and X-ray free electron lasers (FELs), are extending laboratory laser experiments to shorter wavelengths, adding element and chemical state specificity by exciting and probing electronic transitions from core levels. The high pulse energies available ensure that spectroscopies belonging to table top laser, such as, for instance, non-linear optics, can cross the frontier into this new wavelength range. Since the first short wavelength FEL FLASH began operation in Hamburg [1], development has been very rapid. The Linac Coherent Light Source (LCLS) was specified to produce $200 \mathrm{fs}$ pulses, but soon produced few fs pulses [2]. Schemes have been developed to create multi pulse and polychromatic radiation, and FEL light has been used to pump atomic lasers in the X-ray region [3,4]. The XUV/soft X-ray laser FERMI is the first fully coherent FEL facility, as the light possesses the full longitudinal coherence lacking in a pure SASE (Self-Amplified Stimulated Emission) configuration [5]. Coherence is achieved by initiating the FEL process with an external seed. The coherence properties of the seed are indeed transferred to the electron modulation, leading to coherent emission at the undulator resonance and at its harmonics. A similar configuration is adopted in the new facility in China; the Dalian Coherent Light Source (DCLS, Dalian, China) covers the vacuum-ultraviolet (VUV) photon energy range [6]. Advanced schemes of self seeding, both in the soft and hard X-rays, were adopted at LCLS [7] to improve coherence of a SASE FEL. With the development of coherent FEL radiation sources, a new era of X-ray spectroscopy commenced, which may have a comparable impact to that of lasers in optics and spectroscopy [8]. In non-linear ultrafast time-resolved techniques, state-specific information is often provided through multiphoton resonances with combinations of sequential photons. Theoretically, combinations of multiple X-ray photons resonant with core transitions can also characterize different excitation processes due to specific sequences of light-matter interaction. Thus, 
particular sub-processes can be enhanced by matching the pulse frequencies to transitions between molecular Eigen-states. This provides high selectivity and flexibility due to momentum and energy conservation of the interacting photons with the material. The different non-linear processes can typically be ordered by the number of involved photons: sum-frequency generation, two-photon absorption and stimulated emission with two photons, followed by other non-linear X-ray phenomena, time resolved transient gratings (XUV-TG) or four wave mixing (XUV-FWM) spectroscopy (see [8] and references therein). Fluorescent decays in the soft $\mathrm{X}$-ray region allow unique access to the structure of the occupied valence states, while keeping the element selectivity and chemical state specificity of soft X-ray spectroscopies [9]. Unfortunately, the probability for a fluorescent decay in the soft X-ray range is below $1 \%$. With a typical spectrometer acceptance of less than $10^{-5}$ of the full solid angle into which the fluorescence is emitted, soft X-ray emission spectroscopy (XES) or resonant inelastic $X$-ray scattering (RIXS) are experiments where single photons have to be counted for hours in order to obtain useful information. Using stimulated emission spectroscopy combined to high intensity, small bandwidth, tunable soft X-ray beams of different colors, a stimulated beam containing the same information as a fluorescence spectrum can be formed-mitigating the low acceptance angle of the spectrometers, and suppressing the dominating Auger decays that also create electronic damage to the sample. With such techniques, two to three orders of magnitude in the signal levels can be gained through the suppression of Auger processes, while the beam directed into the spectrometer promises to gain about five orders of magnitude in detected signal levels-clearly having the potential to revolutionize how we can study matter with XES or RIXS based spectroscopies [10].

Beyond the understanding of molecular processes, one also wishes to manipulate them. In the quantum mechanical world this can be done by the established methods of coherent control [11,12]. When this approach is developed for FELs, the new frontiers opened up include control of inner valence and core levels, and consequently element sensitivity. Optical coherent control is temporally limited to pulses that are several times the duration of an optical cycle, which is several femtoseconds. In the XUV to $\mathrm{X}$-ray range, the periods are from hundreds to a few attoseconds. Thus, extremely fast processes can be controlled by tuning the relative phases and intensities of pulses delivered to the sample [13].

We provide an overview of the status and perspectives of the FERMI facility as an experimental tool to investigate a large array of phenomena ranging from nano-dynamics in complex materials to phenomena that are at the heart of conversion of light into other forms of energy.

\section{FERMI Overview}

FERMI is located at the Elettra laboratory in Trieste. The FEL facility covers the VUV to soft X-ray photon energy range with two FELs, FEL-1 and FEL-2 both based on the High Gain Harmonic Generation seeded mode (HGHG) [14]. The HGHG scheme consists in preparing the electron beam phase space in a first undulator, called modulator in Figure 1, where the interaction with an external laser, the seed, induces a controlled and periodic modulation in the electron beam longitudinal energy distribution.

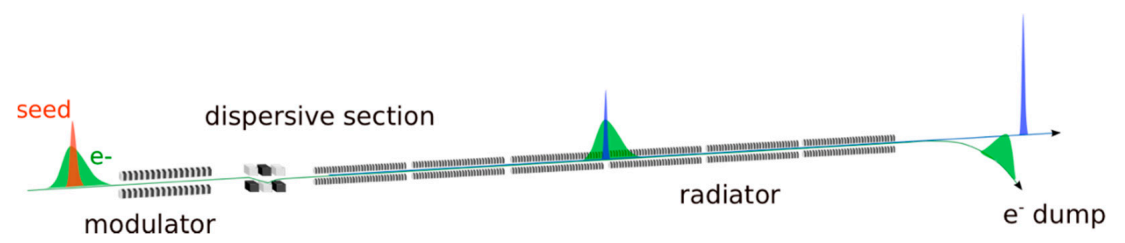

Figure 1. Sketch of the High Gain Harmonic Generation (HGHG) free electron laser (FEL) configuration. Seed laser pulse and electron beam are superimposed in a first undulator indicated as modulator. The FEL interaction induces an electron energy modulation with the periodicity of the seed wavelength. The dispersive section converts this energy modulation into a density modulation containing higher order Fourier components of the original modulation. One of these components is finally amplified in the radiator. 
The beam propagates through a "dispersive section", a magnetic device introducing a strong correlation between the electron energy and the path length. This dispersive section converts the energy modulation into a density modulation, which is characterized by higher order harmonic components and retains the phase properties of the seed. The "density" modulated beam is then injected into a long FEL amplifier, similar to the one adopted in SASE FELs. The amplification process is initially enhanced by the presence of the modulation. The modulation depth is calibrated by tuning the seed intensity, in order to reach FEL saturation and efficient energy extraction at the end of the amplifier.

The HGHG cascade scheme is implemented in FERMI FEL-1, to generate fully coherent radiation pulses in the VUV spectral range [15]. The seed signal, continuously tunable, typically in the range $230-260 \mathrm{~nm}$, is obtained from a sequence of nonlinear harmonic generation and mixing conversion processes from an optical parametric amplifier. The radiation resulting from conversion in the FEL up to the 13th harmonic is routinely delivered to user experiments [16].

The amplitude of the energy modulation necessary to initiate the HGHG process grows with the order of the harmonic conversion. The induced energy dispersion has a detrimental effect on the high gain amplification in the final radiator, for this reason the design of FEL-1 relied on harmonic conversions up to harmonic 13. During the last years of operations, we have demonstrated the ability to operate the FEL at even higher harmonic orders with reduced performances, e.g., up to the 20th harmonic, but substantially higher orders can be reached with a double stage HGHG cascade, where the harmonic conversion is repeated twice. The double conversion is done with the fresh bunch injection technique $[17,18]$ on FERMI FEL-2 [19], shown in Figure 2. The FEL is composed by a first stage, analogous to FEL-1 (see Figure 1), followed by a delay line, a magnetic chicane slowing down the electron beam with respect to the light pulse generated in the first stage. The light pulse from the first stage is shifted to a longitudinal portion of the beam unperturbed by the seed in the first stage. In this way the light from the first stage functions as a short wavelength seed for the second stage. This scheme was implemented for the first time on FERMI FEL-2 and was used to demonstrate the seeded FEL coherent emission in the soft-X rays, up to harmonic orders of 65, and more [19].

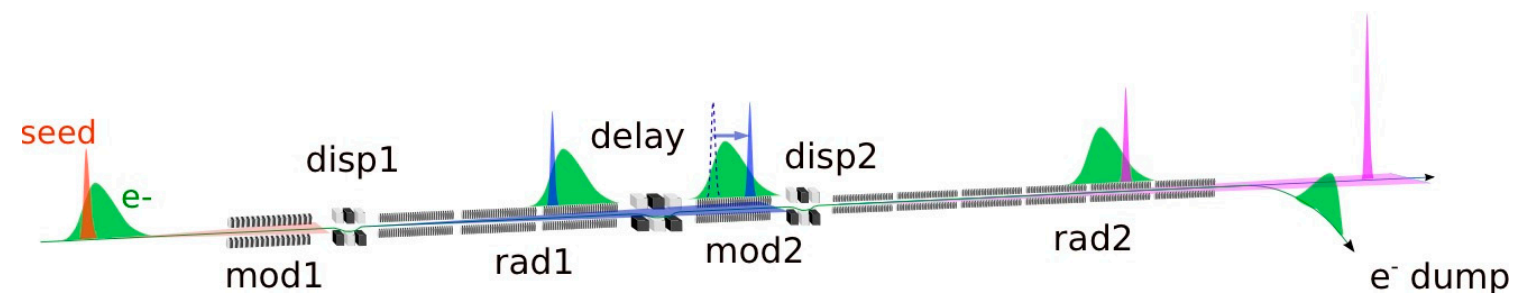

Figure 2. Schematic layout of FERMI (acronym of Free Electron laser Radiation for Multidisciplinary Investigations) FEL-2, implementing the HGHG double stage cascade in the fresh-bunch high-gain harmonic generation configuration. The first stage (mod1-disp1-rad1) is analogous to the high-gain harmonic generation scheme of FEL-1 shown in Figure 1. The second stage (mod2-disp2-rad2) is based on the same concept, but the seed is the radiation produced in the first stage. The two stages are separated by a delay line (delay) which lengthens the electron path with respect to the radiation path allowing to shift the seed over a "fresh" portion of the electron beam.

\section{FEL Performances}

\subsection{Longitudinal Coherence and Pulse Duration}

FEL-1 and FEL-2 may be operated even without the presence of an external seed, i.e., in SASE mode. The emission may be indeed enhanced by tuning the dispersive section of FEL-1 [20] or the multiple dispersion sections of FEL-2 [21], to increase the electron bunching at entrance of the final amplifier. In this configuration, the light pulse resembles the longitudinal structure of the beam current, with a correlation length much shorter than the electron bunch length. The consequence is a longer pulse, of several hundred of fs, with broad spectrum and spiky spectral features, characteristic 
of SASE FELs. This mode of operation can be used in those experiments requiring longer pulses and broadband emission, for alignments, or in the eventuality the seed laser system is not available. In Figure 3 we show the seeded FEL spectrum measured at harmonic order 8 compared to an optimized Optical-Klystron-SASE spectrum (OK-SASE spectrum). In in the example shown in the figure, in seeded mode the relative linewidth drops by about an order of magnitude. Typical relative linewidths of 2 to $5 \times 10^{-4}$ (rms) are routinely achieved on both FEL-1 and FEL-2. Less evident from the images in Figure 3, the presence of the seed also affects the pulse duration.

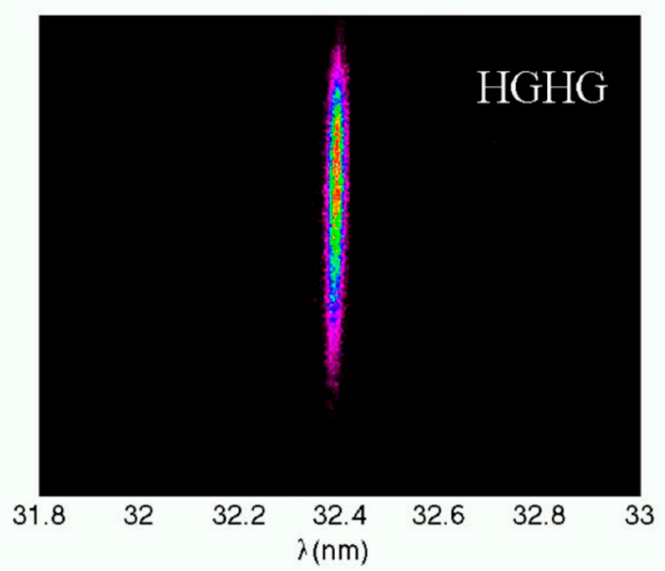

(a)

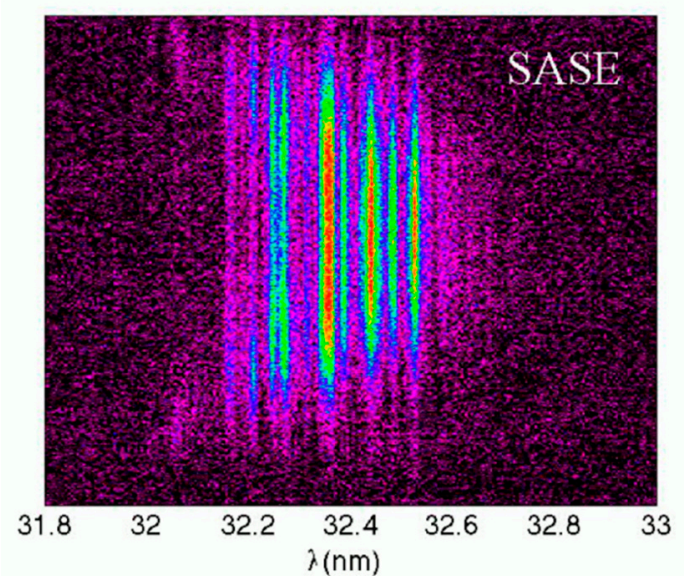

(b)

Figure 3. Spectrum in seeded mode (a) and in Self-Amplified Stimulated Emission (SASE) mode; (b) Wavelengths are dispersed along the horizontal axis. The vertical axis represents the vertical position at the spectrometer detector. The distribution on the vertical axis gives the projection of the beam spatial distribution on the vertical plane.

In seeded mode the pulse duration depends primarily on the duration of the seed, scaled according to the harmonic order $n$ by a coefficient $7 / 6 n^{1 / 3}$ [22]. Typical pulse duration with a seed duration of 120-150 fs are 50-70 fs (fwhm) on FEL-1. FEL-2 seeded with a short seed laser (70 fs) is expected to deliver FEL pulses of about $20 \mathrm{fs}$, depending on the laser setup and harmonic conversion order. We may therefore compare the longitudinal coherence of the source in the two modes of operation, in terms of distance from the Fourier transform limit for a Gaussian pulse (FTL),

$$
\Delta t \Delta \lambda=0.44 \lambda^{2} / c
$$

where $\Delta t$ and $\Delta \lambda$ correspond to the full width half maximum of the temporal and spectral distributions of the optical pulse. If we consider the FERMI SASE conditions of operation in Figure $3 b$ where the spectral width is about $0.3 \mathrm{~nm}$, and where the optical pulse duration should be comparable to the electron bunch length, of the order of $0.5-0.7 \mathrm{ps}$, a rough estimate would indicate the pulse about $120-200 \times$ FTL. This value, with the input seed and with the proper tuning of the FEL parameters, drops to $\approx 1.2-1.5 F T L$ [23]. Specific tuning of the e-beam and the seed laser parameters may be set to bring the FEL even closer to the ideal Fourier limit [24]. The example in Figure 3 corresponds to harmonic order 8 of FEL-1. A narrow, single mode spectral line, such as the one shown in Figure 3 corresponding to harmonic 8 of FEL-1 seeded at $260 \mathrm{~nm}$, is available in the entire spectral window of photon energies emitted by the FERMI FELs ( $20-60 \mathrm{eV}$ on FEL-1 and $60-330 \mathrm{eV}$ on FEL-2). As a second example in Figure 4, the spectrum of the second stage of FEL-2 is shown. The high end of the nominal photon energy range is reached upshifting the seed frequency by harmonic order 13 in the first stage and harmonic 5 in the second stage, for a total frequency upshift of 65. 


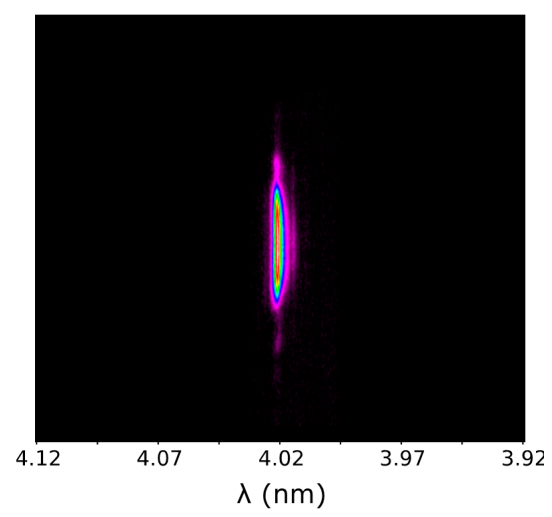

Figure 4. Spectrum of FERMI FEL-2 at harmonic 65 of the input seed. As in Figure 3, wavelengths are dispersed along the horizontal axis. As in Figure 3 the vertical axis represents the vertical position at the spectrometer detector.

\subsection{Energy Per Pulse \& Temporal Jitter}

This dramatic increase of the longitudinal coherence with respect to a SASE source is only the first evident advantage of starting the amplification process from an external seed. The seed introduces indeed a number of additional handles to control amplification and the resulting output pulse properties. The central emission frequency at the lowest order of approximation is determined by the harmonic of the seed laser. The central emission frequency jitter is therefore normally lower than the linewidth by up to an order of magnitude. A SASE source is characterized by an intrinsic pulse energy fluctuation associated with the startup from the electron shot noise. From this point of view a seeded FEL is, conversely, a completely deterministic system.

The fluctuations of the pulse energy are determined by fluctuations of the external parameters driving the amplifier, as the energy of the electron beam, the timing between electrons and seed, and the intensity of the seed itself. The energy jitter may be lower than $5 \%$, as in the example shown in Figure 5, where the histogram of the energy per pulse from FEL-1 in optimized conditions at $51 \mathrm{~nm}$ is displayed. This value may be compared to the typical energy jitter of the input seed laser in the UV, which is about 1\%. A typical figure for the energy jitter is 10-15\% for FEL-1 and 25-35\% for FEL-2 with the double cascade. In terms of temporal synchronization, the seed timing largely determines the arrival time of the FEL photon pulse. At FERMI the optical synchronization between the seed and the lasers used in pump an probe experiments, which originates from the same oscillator, is associated to a jitter between the FEL light and the other sources of about 5 fs [25]. Recent measurements have shown even lower values, below 3 fs [22].

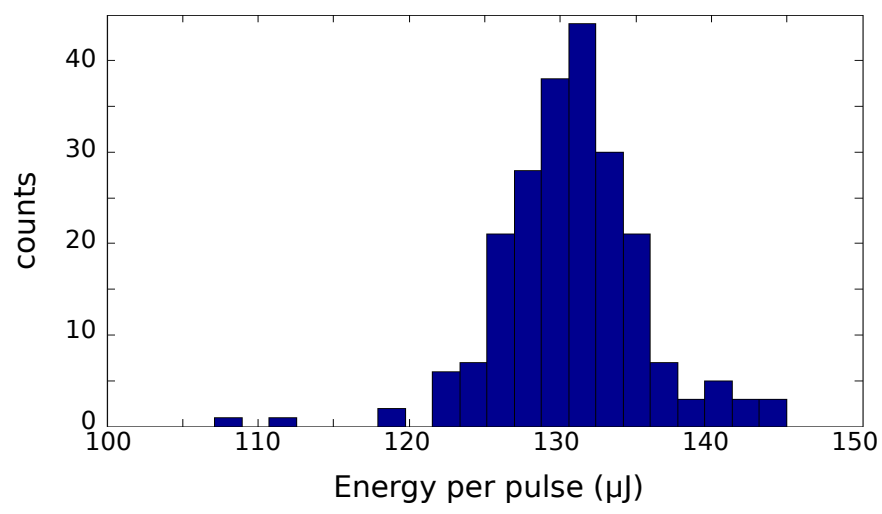

Figure 5. Histogram of the energy per pulse measured from FEL-1 at harmonic $5(51 \mathrm{~nm})$. Acquisition of 220 consecutive shots, average $131 \mu \mathrm{J}$, standard deviation $4.8 \mu \mathrm{J}$. 
The typical energy per pulse depends on the wavelength of operation and in the requirements on the spectral linewidth. Highest energy per pulse may be achieved in saturated conditions, where the pulse shape may be affected by non-linear processes in the longitudinal electron dynamics. On FEL-1 an energy per pulse larger than $100 \mu \mathrm{J}$ is available in the entire range of operation (20-100 nm), in conditions of single mode, narrow linewidth spectral line. On FEL-2 this is possible in the low end of the spectral energy range, at wavelengths above $8-10 \mathrm{~nm}$. The average energy per pulse decreases while increasing the photon energy, down to about $10 \mu \mathrm{J}$ at $4 \mathrm{~nm}$. The energy per pulse may be lower than the one achievable in an optimized equivalent SASE FEL where most of the charge in the bunch participates to the FEL action. The seeded portion of the electron beam is typically of the order of $100 \mathrm{fs}$, which can be $10-20 \%$ or less of the entire bunch. Compressing the entire electron bunch down to the seed duration in an ultrashort multi-kA spike of current would ensure a higher efficiency, but would also introduce a much higher sensitivity to longitudinal jitter between the seed pulse and the current, and the compression process would be strongly affected by self-field effects, such as space charge, coherent synchrotron radiation and space charge fields [26], which alter the smooth distribution of the electron longitudinal phase space necessary to preserve the quality of the pulse during amplification. Moreover, an excess of peak current would increase the gain to a value leading the electron shot noise background to compete with the seed at saturation, and this would remove most of the advantages of seeding the amplifier. An alternative to extend the seeded region of the electron current is to use a temporally stretched, chirped seed pulse, thus extracting a larger amount of energy from the electrons. The chirp properties of the output pulse are strictly correlated to those of the seed. The FEL pulse can be therefore optically compressed to increase the peak power. The scheme is equivalent to the chirped pulse amplification scheme (CPA), commonly adopted in ultrashort pulse-solid state lasers [27]. Here the issue is not that of reducing the peak power in an active medium, but to allow the use of an extended electron beam longitudinal region, increasing the active bunch charge, without compressing the electrons to the ultimate limit. Chirped pulse amplification (CPA) in FEL amplifiers was proposed originally in [28] and further studied in the framework of a SASE amplifier in [29]. The scheme was later demonstrated at FERMI in [30].

\subsection{Multiple Pulses}

The intensity of the seed determines the depth level of saturation reached in the amplifier. This parameter can be tuned to control the intensity and to some extent the longitudinal shape of the laser pulse. An excess of seed can indeed be used for the generation of virtually jitter-free twin pulses delayed in time [31], which can also be separated in frequency by a proper frequency chirp of the input seed $[32,33]$. There are several other methods for generating multiple color-multiple pulses at FERMI. From FEL-2 for example, two color pulses almost superimposed in time, are naturally available as the emission from the first stage and the second stage. In normal operation, the light from the first stage has to be removed or attenuated by gas attenuators or filters, but in some specific experiments the VUV light from the first stage can be used in combination with XUV-Soft-X ray emitted by the second stage. This setup was used in the study of the ultrafast dynamic of melting in Si monitored by tracking the L(2,3)-edge shift [34]. Multiple pulses of different colors can be also generated on FEL-1. In Figure 6 we sketch three methods which were demonstrated in the past. In Figure $6 \mathrm{a}$ a second color is obtained by simply tuning part of the radiator at one harmonic and part of it at a second, different harmonic. The temporal jitter between the two components was estimated in an experiment of coherent control where we manipulated the relative phase of two colors $(63.0 \mathrm{~nm}$ and $31.5 \mathrm{~nm})$ to control the asymmetry of the photoelectron angular distribution of ionized neon with a temporal resolution of 3 as [13]. Temporally separated pulses can be generated in the setup of Figure 6b. A double seed can be indeed injected with a temporal delay between the two seed pulses, with delays comprised between 200 and 600-700 fs, to ensure overlapping with the electron beam current. Even in this case the temporal jitter between the two pulses can be sufficiently low to preserve a phase locking between the two pulses [35]. The two seed pulses can be also separated in frequency to generate two distinguishable colors, with 
the condition that they are both included in the gain bandwidth of the FEL amplifier (0.7-0.8\%) [36]. A larger frequency separation between the two pulses can be achieved (Figure 6c) if the amplifier is tuned to different harmonics of the seed. In this case two seed pulses are still separated in frequency by less than the bandwidth of the modulator ( $3 \%$ ) and both generate energy, and then density modulation at the entrance of the amplifier. The amplifier is then separated in two parts, one resonant with the modulation resulting from the first seed pulse and the other resonant with the one from the second. The two amplified pulses have to be separated in frequency to be generated and amplified separately in these two undulator parts and the amplification can happen on different harmonics of the seed [37].

a)

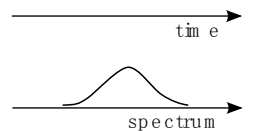

b)

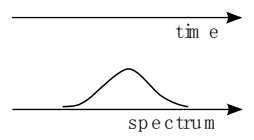

c)

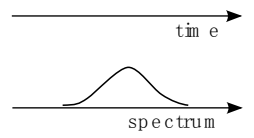

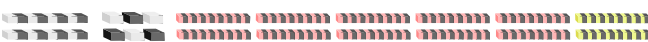

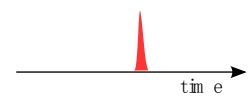

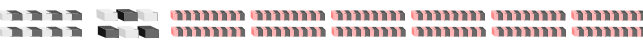
ดั

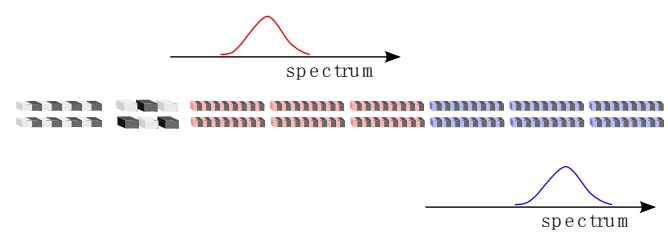

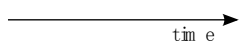

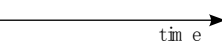

Figure 6. Various method for the generation of multiple pulses adopted on FERMI FEL-1. (a) Two colors can be generated by tuning the final amplifier to different harmonics of the seed. The output pulse is composed by the superposition of the two harmonic components; (b) A double seed can be injected with a temporal delay between the two seed pulses comprised between 200 and 600-700 fs. The two seed pulses can be separated in frequency to generate two distinguishable colors, by ensuring they are both included in the gain bandwidth of the FEL amplifier (0.7-0.8\%); (c) A larger frequency separation between the two pulses is also possible if the amplifier is tuned to different harmonics of the seed.

\section{XUV Wave Mixing and Coherent Control}

In this section, we discuss the advantages offered by a fully coherent FEL source in the extension of experimental techniques as non-linear optics at shorter wavelength. An interesting aspect of the development of non-linear optics is that the main concepts at the basis of this field were already available for J. C. Maxwell (if in 1861 he had considered a power series expansion in the Maxwell equations) and $\mathrm{H}$. A. Lorentz (if in 1878 he had introduced anharmonic terms in the oscillator model of the atom). The point is that neither Maxwell nor Lorentz had the experimental tools for inspiring their research in this direction. Until a few years ago non-linear light-matter interactions in the extreme ultraviolet (XUV) and X-ray range were basically ignored for the same reason. However nowadays FELs have allowed undertaking relevant steps towards the exploitation of XUV / X-ray non-linear optics. For instance, FELs have been employed to demonstrate: stimulated X-ray emission [3], amplified spontaneous XUV emission [10], X-ray-optical sum-frequency generation [38], X-ray two-photon absorption [39], X-ray second harmonic generation [40]; all these achievements were gained in the last few years, reflecting rapid growth in the field. A common motivation for many of the aforementioned investigations is the outlook represented by XUV/X-ray wave mixing experiments. 


\subsection{Four Wave Mixing}

Among wave-mixing processes, four wave mixing (FWM) processes have a special place, since they are at the basis of most experimental methods, besides being the lowest order non-linear processes that are not vanishing by reason of symmetry. FWM arise from the 3rd-order non-linear interactions of three coherent electromagnetic fields $\left(E_{1,2,3}\right)$ that might have different frequencies $\left(\omega_{1,2,3}\right)$, wavevectors $\left(k_{1,2,3}\right)$, polarizations, bandwidth, time delays, etc. (see Figure 7$)$.

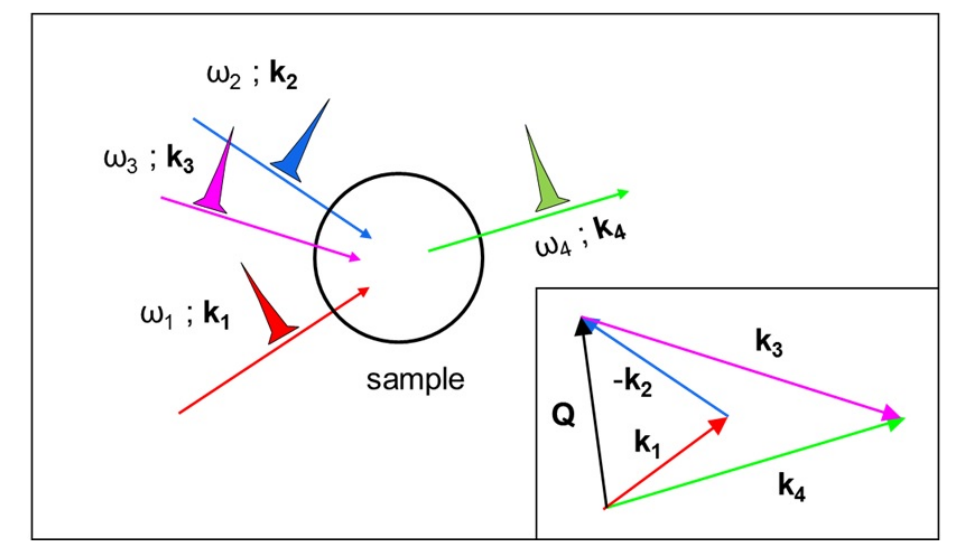

Figure 7. Sketch of the four wave mixing (FWM) experimental scheme. After the full stop: The non-linear interaction of the three photon pulses (i.e., coherent electromagnetic fields $E_{1,2,3}$ ) that may have different frequencies and wavevectors $(1,2,3)$ is represented in the figure. The pulses may have as well different polarization, bandwidth and time delays. In the inset is depicted the phase matching condition.

Such interacting fields drive the radiation of a fourth (signal) field, whose photon parameters (frequency, $\omega_{4}$, wavevector, $k_{4}$, polarization, etc.) may differ from those of the input fields. The possibility to experimentally control the input field parameters and to determine the output field parameters turns into the capability to stimulate and detect different FWM processes, which often carry out distinct and complementary information on the sample under study. Such a high degree of selectivity and richness of information makes FWM an extremely versatile and informative tool. For instance, through a FWM process termed impulsive stimulated scattering (ISS) it is possible to determine in real time and with wavevector selectivity the dynamics of both ultrafast (sub-picosecond) molecular vibrations or slow (millisecond) thermal and structural relaxations, depending on the bandwidth and time delays of the interacting fields [41]. Information on the dynamics of higher energy (i.e., faster) sample excitations can be achieved, e.g., by coherent Raman scattering (CRS) and coherent multi-dimensional spectroscopy; methods also able to provide insights into the correlations between different dynamical variables, such as those related to vibrational and electronic modes [42]. FWM approaches are also bringing relevant technological advances, ranging from sub-wavelength microscopy [43] to wavefunction tomography during chemical reactions [44]. The hard-limit of optical FWM, as any other optical spectroscopy, is inherently related to the long wavelength (some 100's of $\mathrm{nm}$ or more) and low photon energy (a few eV's or less) of the optical radiation itself. This prevents to probe matter at atomic and molecular length scales (a few nm or less) as well as to study excitations with energies larger than a few $\mathrm{eV}^{\prime} \mathrm{s}$, a limitation that basically confines optical CRS to the study of vibrational dynamics and that of low-energy electronic excitations. Furthermore, optical photons cannot exploit core-level electronic resonances, typically located in the 10 's eV to 10 's keV range, adding elemental selectivity to the FWM approach. The latter point combined with the possibility to detect high energy excitation (e.g., valence band excitons in the 1-10 eV energy range) on a Q-range comparable with the inverse molecular size $\left(\sim \mathrm{nm}^{-1}\right)$ are the main advantages expected from the XUV / X-ray analogue of CRS (XCRS). By tuning the frequencies of the input beams involved in the 
XCRS process to core resonance of distinct atoms it would be possible to determine where a given electronic wave-packet is created and where it is probed, as well as to follow in real time the dynamics of such charge-transfer between the selected atoms. This unique capability arises from the multi-wave nature of XCRS, which permits to overcome the basic limitation of any linear X-ray method, where the light-matter interaction occurs in correspondence of a single atomic site and prevents the detection of real-time dynamics between distinct atoms. Moreover, XCRS is not limited to the study of valence band excitons, since the same concept in principle applies for all kind of excitations, related to any dynamical variable coupled to the field (in some circumstances also to those uncoupled in the linear regime), with energies lower than that of core-resonances. Such class basically includes all kind of modes related to nuclear (phonons, structural relaxations, heat diffusion, etc.), electronic (excitons, plasmons, etc.) and "mixed" (polarons, polaritons, etc.) degrees of freedom. The XCRS approach may hence allow to study, e.g., charge and energy transfer processes among different atoms in molecules, the delocalization and correlations of electronic excitations as well as structural fluctuations, nuclear motions and relaxation processes, other than detecting the dynamics of elementary excitations such as, e.g., phonons, plasmons and polarons. Among the worldwide existing FEL facilities, FERMI possesses unique characteristics for FWM experiments in the XUV/soft X-ray domain. Firstly, most of the photon parameters of the FEL radiation emitted (the longitudinal coherence properties among them) are related to those of the seed laser, which is fully controllable in all relevant parameters, so that it is possible to control the FEL output by simply acting on the seed. Moreover, it is possible to use two (or more) independently controllable seed laser pulses, which result into the radiation of two (or more) FEL-pulses with controllable photon energy, polarization, time delay, etc.; a further development of such scheme includes the simultaneous use of multiple resonances in the FEL amplifier, which largely extends the separation in the photon energy (i.e., up to several $\mathrm{eV}^{\prime} \mathrm{s}$ ) of the multiple FEL pulses [37]. Finally, the layout of FERMI could be adapted to generate a few fs to sub-fs FEL-pulses with the aforementioned benefits. In a nutshell, today FERMI is the FEL source most similar to a "multi-color" conventional laser and, therefore, it could be used to attempt the development of XUV/soft X-ray FWM. A significant endorsement of the latter statement is represented by the recent demonstration of a time-resolved FEL-stimulated FWM response [45] (see Figure 8). In this experiment two time-space coincident ultrafast (time duration $\sim 70 \mathrm{fs}$ ) FEL pulses (photon energy $\omega_{\mathrm{XUV}} \sim 45 \mathrm{eV}$ ) were crossed at the sample (amorphous $\mathrm{SiO}_{2}$ ) position.

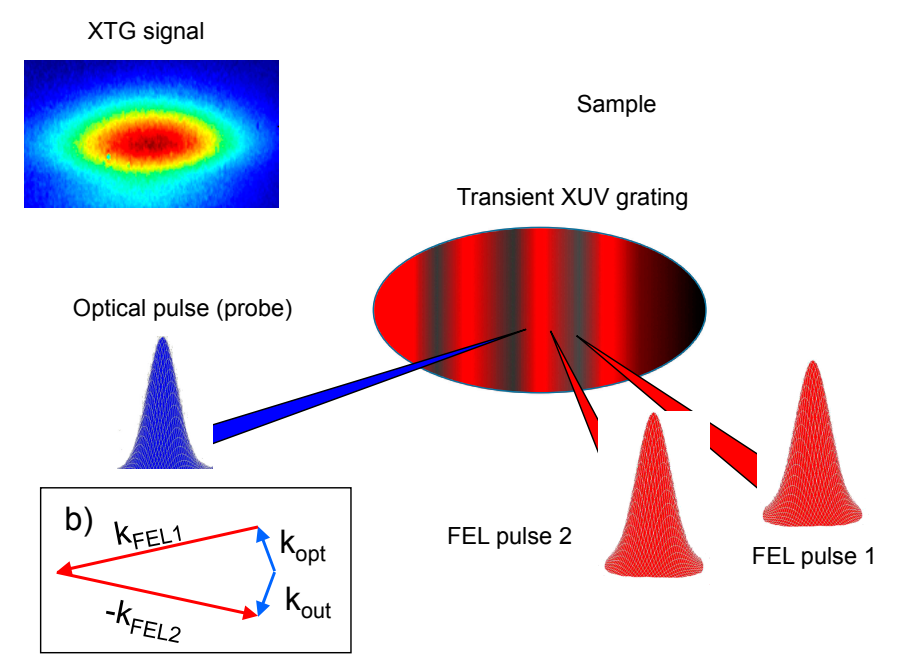

Figure 8. FEL-based FWM experiment stimulated by transient extreme ultraviolet (XUV) gratings (inset (b) reports the phase matching geometry: $k_{\mathrm{FEL} 1}, k_{\mathrm{FEL} 2}, k_{\mathrm{opt}}$ and $k_{\mathrm{out}}$ are the wavevectors of the two FEL pulses, the optical pulse and the FWM signal, respectively). The signal (XTG) has been registered on a charged coupled detector (CCD). 
These beams were expected to generate an XUV grating able to scatter off, through FWM processes, a third coherent beam, provided that the latter is send into the sample in phase matching conditions. Indeed, using a third "phase matched" optical pulse (time duration $~ 100 \mathrm{fs}$, photon energy $\omega_{\text {opt }} \sim 3.1 \mathrm{eV}$ ) in the interaction region allowed the observation of an optical beam emerging from the sample along the expected "phase matched" direction (see Figure 8 XTG signal).

\subsection{Perspective for the XUV/X-ray FWM}

Further steps can be undertaken to make XUV/X-ray FWM experiments feasible on routine basis. One of the most useful would be to replace the optical pulse with a XUV/X-ray. This will permit to probe, via ISS-type FWM methods, low energy modes (e.g., acoustic modes) in a wavevector range $\left(\sim 0.1-1 \mathrm{~nm}^{-1}\right)$ hardly accessible by both optical methods and linear X-ray spectroscopy [46]. Such a wavevector range matches the characteristic length-scales of heterogeneities in the local structures of several classes of materials (e.g., block copolymer, relaxor ferroelectrics, glasses etc.), the incommensurate dimensions of many crystalline phases showing super-lattices of different natures, as well as the characteristic dimensions and periodicities of many nanostructures. Among these applications we mention the study of acoustic dynamics in glasses, which is largely believed to be the origin of the anomalous thermal properties of glasses, still a lively debated issue [47]. A debate that essentially comes from the impossibility to fully access such a $\sim 0.1-1 \mathrm{~nm}^{-1}$ wavevector range and from the stark disagreement often observed between the extrapolations of the trends found at lower and larger wavevectors. Also, couplings between acoustic modes and local vibrations in nm-sized elastic domains, inherently connected to the amorphous local structure, may also have a relevant role; such a small length-scale is potentially in the range of XUV/X-ray FWM. Another relevant step to extend the range of applications of FEL-based FWM is to exploit the two-color emission scheme in order to demonstrate FEL-based CRS-type experiments. This would be a major step forward towards the realization of XCRS with atomic selectivity (see Figure 9), which in many respects may be considered as a main target application of XUV/X-ray FWM.

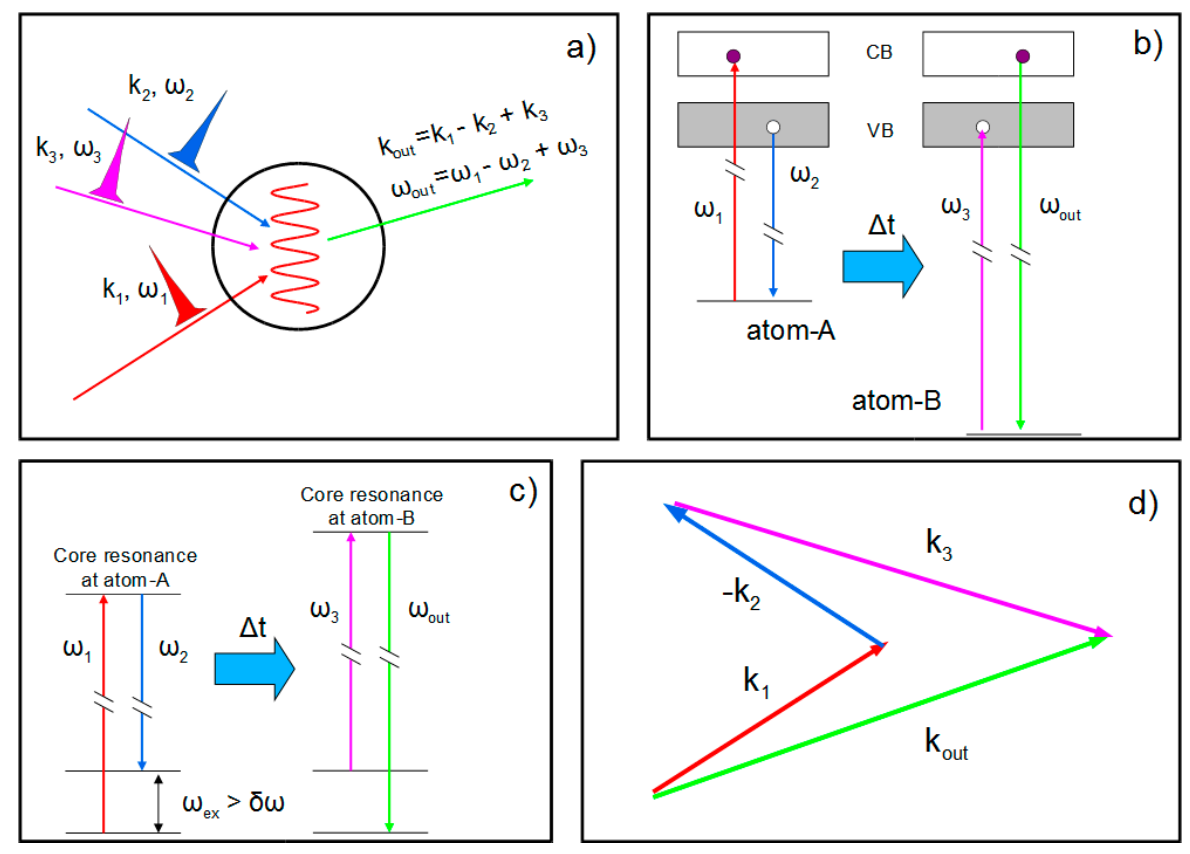

Figure 9. (a) Sketch of the FEL-based X-ray analogue of coherent Raman scattering (XCRS) experiment; $(\mathbf{b}, \mathbf{c})$ are the excitation processes and level scheme for a XUV / X-ray CRS experiment involving core transitions in both the excitation and probing process; (d) Phase matching diagram. 
Indeed, the potential of XCRS to follow charge and energy flows between constituent atoms in materials would be of the greatest importance to address some fundamental scientific issues, such as: (a) the study of intramolecular relaxation dynamics in metal complexes, which are the doorway to photo-induced charge separation (the high selectivity of XCRS would be exploited to understand and disentangle processes, such as intramolecular vibrational redistribution, internal conversion and intersystem crossing, occurring upon photo-excitation; those are processes of fundamental interest in devising efficient molecular systems for applications as diverse as solar energy conversion, biology or data storage [48]); and (b) the dynamics of charge injection and transport in photocatalytic reactions taking place in metal oxides nanoparticles, such as $\mathrm{TiO}_{2}$, that are key materials for renewable energy; for instance, the key event occurring in devices for solar energy conversion is the generation of a charge-separated state through ultrafast electron injection from an excited metal-complex, adsorbed on a nanoporous metal oxide substrate, to the conduction band of the substrate [49]. In this context the atomic selectivity of XCRS may allow us to understand whether such ultrafast electronic excited states have an $\mathrm{O}$ or Ti character.

\subsection{Coherent Control}

In order to manipulate molecular processes, coherent control could be the technique of choice. In this kind of experiment Phase coherent light of one or more colours interacts with a target. The outcome of the interaction is determined by the phase and amplitude of the light. Ion yield, direction of emission of ions and electrons, and so on, can be controlled. In the XUV to X-ray range, the times of the duration of an optical cycle periods are from hundreds to a few attoseconds thus increasing the temporal resolution of orders of magnitude when comparing to optical lasers. Recently FERMI has been employed to carry out the first XUV coherent control experiment where the first and the second harmonics were ionizing Neon on the $2 p^{5} 4 s$ resonance [13]. While the second harmonic was producing a single photon ionization process, the first was exiting two photon ionization processes (see Figure 10a).
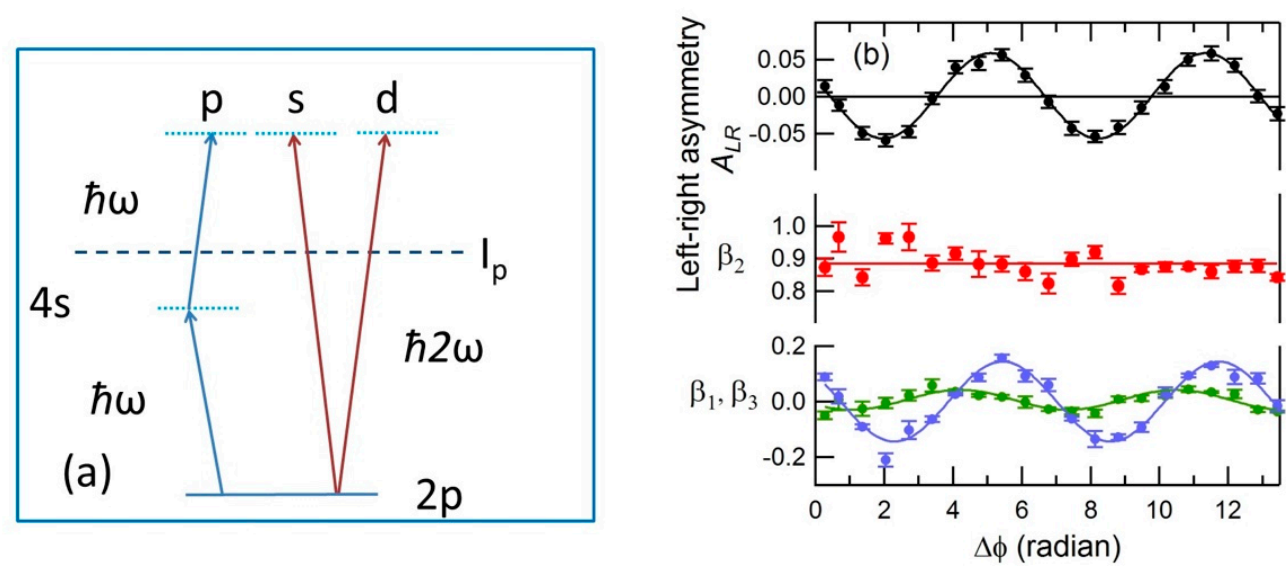

Figure 10. (a) Scheme of the experiment. Left: a $2 p$ electron is excited to $4 s$ by one photon and then emitted as a $p$-wave by a second photon. Right: a $2 p$ electron process is emitted as an $(s+d)$-wave by a one-photon process. (b) Asymmetry parameter $A_{L R}$ as a function of $\Delta \phi$ (black curve), and $\beta_{1}$ (green), $\beta_{3}$ (blue) and $\beta_{2}$ (red) parameters as a function of $\Delta \phi$. Experimental data are shown as markers with error bars. The lines are sinusoidal or straight line fits for $\beta_{1}, \beta_{3}$, and $\beta_{2}$ respectively.

The possibility offered by FERMI to change the phase among the two harmonics (see Figure 10b) allowed the first experimental demonstration that coherent control experiments can be carried out in the XUV X-ray region.

Much effort is being invested to develop ever shorter pulses at FELs. The arrival of phase control of multi-color pulses now means that they can be designed to produce trains of very short pulses, since 
a train of attosecond pulses can be constructed from a coherent superposition of coherent femtosecond pulses with commensurate wavelengths. This ability to tailor pulses may eventually lead to "design" single pulses of attosecond duration.

\section{Discussion on Future Perspectives}

A number of critical developments would boost the impact of the FERMI FELs in nonlinear multi-wave spectroscopies and the other applications of the seeded FERMI FEL sources. The Elettra-Sincrotrone Trieste scientific advisory committee, has recently outlined the importance of extending the spectral range of FEL-2 toward higher photon energies, that would allow to reach the K-edges of $\mathrm{C}(284 \mathrm{eV}), \mathrm{N}(410 \mathrm{eV})$ and $\mathrm{O}(543 \mathrm{eV})$. Higher order harmonics could be exploited to extend the photon delivery to the L-edges of the $3 \mathrm{~d}$ transition metals relevant for magnetism catalysis and solar energy production. The required increase in electron beam energy would be modest and within the capabilities of the present infrastructure. On the other side, the generation of shorter pulses (sub-10 fs) would allow the investigation of the faster electron dynamics at the level of typical core hole lifetimes ( $4-8 \mathrm{fs}$ for $\mathrm{O}, \mathrm{N}$ and $\mathrm{C}$ ). We already mentioned the chirped pulse amplification as a means for the generation of ultrashort pulses. Other options are under investigation to reduce the pulse duration while maintaining the synchronization and longitudinal coherence properties of the seeded source. On one side a proper shaping of the beam properties could limit the longitudinal region where lasing takes place [50], and the FERMI linac laser heater is an additional handle to control the properties of the FEL light [51,52]. On the other side, nonlinear dynamics at saturation may lead to a longitudinal self-focusing regime [53-55] which is well suited for reaching the sub-10 fs regime in the FERMI configuration. A second direction for the future development of FERMI in multidimensional spectroscopy and the control of the light properties in advanced pump and probe configurations is in a further extension of the flexibility for the generation of multiple colour, multiple pulses. While the generation of multiple pulses on FEL-1, with the constraints imposed by the double lasing condition, is routinely achieved, it is all but straightforward in the higher photon energy range, on the double stage configuration of FEL-2. The same spectral range could be reached on FEL-2 with an Echo Enabled harmonic generation configuration (EEHG) [56-58] that would allow on FEL-2 a similar flexibility as the one demonstrated on FEL-1. For this purpose, an experiment on FEL-2 is under study [59] and is planned for 2018. The requirement for different pump and probe pulses even calls for frequency tripling for the few-fs FEL emission scheme. In this respect, the possibility of combining FEL1 and FEL2 on the same experiment, with a variable controlled delay, could lead to a further extension in multicolour multiple pulses generation. The coherence properties of FEL-1 and FEL-2 could be combined while maintaining higher pulse energy and the full FELs flexibility in the choice of the radiation colour for the two pulses [60].

\section{Conclusions}

Free electron laser radiation, through its unique combination of parameters, namely ultrashort pulses with high brightness and coherence, has led to revolutionary steps forward in time resolved X-ray methodologies. A decade ago these developments were just dreams for the researchers who have recognized that a broad class of phenomena can be investigated by ultrafast $X$-rays [61]. The field of non-linear XUV /X-ray optics is one of the youngest within fields opened by FELs and we expect that it will advance fast, paralleling the ongoing development of FEL technology, and will contribute to gaining a deeper comprehension of fast dynamics and learning how to manipulate materials. In this framework, the FERMI facility would play a major role, thanks to its unique characteristics related to the pioneering laser-seeding scheme.

Acknowledgments: The authors gratefully acknowledge the FERMI teams for the outstanding work done in running the facility and for kindly providing the reference data and pictures on the FEL operation reported in this document.

Author Contributions: L.G. and C.M. equally contributed to the preparation of text and figures of this manuscript. 
Conflicts of Interest: The authors declare no conflict of interest.

\section{References}

1. Tiedtke, K.; Azima, A.; von Bargen, N.; Bittner, L.; Bonfigt, S.; Düsterer, S.; Faatz, B.; Frühling, U.; Gensch, M.; Gerth, C.; et al. The soft X-ray free-electron laser FLASH at DESY: Beamlines, diagnostics and end-stations. New J. Phys. 2009, 11, 023029. [CrossRef]

2. Emma, P.; Akre, R.; Arthur, J.; Bionta, R.; Bostedt, C.; Bozek, J.; Brachmann, A.; Bucksbaum, P.; Coffee, R.; Decker, F.J.; et al. First lasing and operation of an angstrom-wavelength free-electron laser. Nat. Photonics 2010, 4, 641-647. [CrossRef]

3. Rohringer, N.; Ryan, D.; London, R.A.; Purvis, M.; Albert, F.; Dunn, J.; Bozek, J.D.; Bostedt, C.; Graf, A.; Hill, R.; et al. Atomic inner-shell X-ray laser at 1.46 nanometres pumped by an X-ray free-electron laser. Nature 2012, 481, 488-491. [CrossRef] [PubMed]

4. Yoneda, H.; Inubushi, Y.; Nagamine, K.; Michine, Y.; Ohashi, H.; Yumoto, H.; Yamauchi, K.; Mimura, H.; Kitamura, H.; Katayama, T.; et al. Atomic inner-shell laser at 1.5-ångström wavelength pumped by an X-ray free-electron laser. Nature 2015, 524, 446-449. [CrossRef] [PubMed]

5. Allaria, E.; Badano, L.; Bassanese, S.; Capotondi, F.; Castronovo, D.; Cinquegrana, P.; Danailov, M.B.; D'Auria, G.; Demidovich, A.; De Monte, R.; et al. The FERMI free-electron lasers. J. Synchrotron Radiat. 2015, 22, 485-491. [CrossRef] [PubMed]

6. Nannan, Z. Dalian Coherent Light Source Produces Laser Output for the First Time. Available online: http: //english.cas.cn/newsroom/research_news/201610/t20161013_168621.shtml (accessed on 12 May 2017).

7. Amann, J.; Berg, W.; Blank, V.; Decker, F.-J.; Ding, Y.; Emma, P.; Feng, Y.; Frisch, J.; Fritz, D.; Hastings, J.; et al. Demonstration of self-seeding in a hard-X-ray free-electron laser. Nat. Photonics 2012, 6, 693-698. [CrossRef]

8. Bencivenga, F.; Capotondi, E.; Kiskinova, M.; Masciovecchio, C. Coherent and transient states studied with X-ray FELs: Present and future prospects. Adv. Phys. 2015, 63, 327-404. [CrossRef]

9. Nordgren, J.; Bray, G.; Cramm, S.; Nyholm, R.; Rubensson, J.E.; Wassdahl, N. Soft X-ray Emission Spectroscopy Using Monochromatized Synchrotron Radiation (Invited). Rev. Sci. Inst. 1989, 60, 1690. [CrossRef]

10. Beye, M.; Schreck, S.; Sorgenfrei, F.; Trabant, C.; Pontius, N.; Schüßler-Langeheine, C.; Wurth, W.; Föhlisch, A. Stimulated X-ray Emission for Materials Science. Nature 2013, 501, 191. [CrossRef] [PubMed]

11. Brif, C.; Chakrabarti, R.; Rabitz, H. Control of quantum phenomena: Past, present and future. New J. Phys. 2010, 12, 075008. [CrossRef]

12. Ehlotzky, F. Atomic phenomena in bichromatic laser fields. Phys. Rep. 2001, 345, 175-264. [CrossRef]

13. Prince, K.C.; Allaria, E.; Callegari, C.; Cucini, R.; de Ninno, G.; Di Mitri, S.; Diviacco, B.; Ferrari, E.; Finetti, P.; Gauthier, D.; et al. Coherent control with a short-wavelength Free Electron Laser. Nat. Photonics 2016, 10, 176-179. [CrossRef]

14. Yu, L.H. Generation of intense UV radiation by subharmonically seeded single-pass free-electron lasers. Phys. Rev. A 1991, 44, 5178-5193. [CrossRef] [PubMed]

15. Allaria, E.; Appio, R.; Badano, L.; Barletta, W.A.; Bassanese, S.; Biedron, S.G.; Borga, A.; Busetto, E.; Castronovo, D.; Cinquegrana, P.; et al. Highly coherent and stable pulses from the FERMI seeded free-electron laser in the extreme ultraviolet. Nat. Photonics 2012, 6, 699-704. [CrossRef]

16. Allaria, E.; Battistoni, A.; Bencivenga, F.; Borghes, R.; Callegari, C.; Capotondi, F.; Castronovo, D.; Cinquegrana, P.; Cocco, D.; Coreno, M.; et al. Tunability experiments at the FERMI@Elettra free-electron laser. New J. Phys. 2012, 14, 113009.

17. Ben-Zvi, I.; Yang, K.M.; Yu, L.H. The "fresh-bunch" technique in FELS. Nucl. Instrum. Methods Phys. Res. A 1992, 318, 726-729. [CrossRef]

18. Yu, L.H.; Ben-Zvi, I. High-gain harmonic generation of soft X-rays with the "fresh bunch" technique. Nucl. Instrum. Methods Phys. Res. A 1997, 393, 96-99. [CrossRef]

19. Allaria, E.; Castronovo, D.; Cinquegrana, P.; Craievich, P.; Dal Forno, M.; Danailov, M.B.; D’Auria, G.; Demidovich, A.; de Ninno, G.; Di Mitri, S.; et al. Two-stage seeded soft-X-ray free-electron laser. Nat. Photonics 2013, 7, 913-917. [CrossRef] 
20. Penco, G.; Allaria, E.; de Ninno, G.; Ferrari, E.; Giannessi, L. Experimental Demonstration of Enhanced Self-Amplified Spontaneous Emission by an Optical Klystron. Phys. Rev. Lett. 2015, 114, 013901. [CrossRef] [PubMed]

21. Penco, G.; Allaria, E.; de Ninno, G.; Ferrari, E.; Giannessi, L.; Roussel, E.; Spampinati, S. Optical Klystron Enhancement to Self-Amplified Spontaneous Emission at FERMI. Photonics 2017, 4, 15. [CrossRef]

22. Finetti, P.; Höppner, H.; Allaria, E.; Callegari, C.; Capotondi, F.; Cinquegrana, P.; Coreno, M.; Cucini, R.; Danailov, M.B.; Demidovich, A.; et al. Pulse duration of seeded free-electron lasers. Phys. Rev. X 2017, 7, 021043. [CrossRef]

23. De Ninno, G.; Gauthier, D.; Mahieu, B.; Rebernik, P.; Allaria, E.; Cinquegrana, P.; Danailov, M.B.; Demidovich, A.; Ferrari, E.; Giannessi, L.; et al. Single-shot spectro-temporal characterization of XUV pulses from a seeded free-electron laser. Nat. Commun. 2015, 6, 8075. [CrossRef] [PubMed]

24. Gauthier, D.; Ribič, P.R.; De Ninno, G.; Allaria, E.; Cinquegrana, P.; Danailov, M.B.; Demidovich, A.; Ferrari, E.; Giannessi, L.; Mahieu, N.; et al. Spectro-temporal shaping of free-electron laser pulses. Phys. Rev. Lett. 2015, 115, 114801. [CrossRef] [PubMed]

25. Danailov, M.B.; Bencivenga, F.; Capotondi, F.; Casolari, F.; Cinquegrana, P.; Demidovich, A.; Giangrisostomi, E.; Kiskinova, M.P.; Kurdi, G.; Manfredda, M.; et al. Towards jitter-free pump-probe measurements at seeded free electron laser facilities. Opt. Express 2014, 22, 12869-12879. [CrossRef] [PubMed]

26. Giannessi, L. Simulation codes for high brightness electron beam free-electron laser experiments. Phys. Rev. Spec. Top. Accel. Beams 2003, 11, 114802. [CrossRef]

27. Strickland, D.; Mourou, G. Compression of amplified chirped optical pulses. Opt. Commun. 1985, 56, 219. [CrossRef]

28. Yu, L.H.; Johnson, D.; Li, D.; Umstadter, D. Femtosecond free-electron laser by chirped pulse amplification. Phys. Rev. E 1994, 49, 4480-4486.

29. Frassetto, F.; Giannessi, L.; Poletto, L. Compression of XUV FEL pulses in the few-femtosecond regime. Nucl. Instrum. Methods Phys. Res. Sect. A 2008, 593, 14-16. [CrossRef]

30. Gauthier, D.; Allaria, E.; Coreno, M.; Cudin, I.; Dacasa, H.; Danailov, M.B.; Demidovich, A.; Di Mitri, S.; Diviacco, B.; Ferrari, E.; et al. Chirped pulse amplification in an extreme-ultraviolet free-electron laser. Nat. Commun. 2016, 7, 13688. [CrossRef] [PubMed]

31. Labat, M.; Joly, N.; Bielawski, S.; Szwaj, C.; Bruni, C.; Couprie, M.E. Pulse splitting in short wavelength seeded free electron lasers. Phys. Rev. Lett. 2009, 103, 264801. [CrossRef] [PubMed]

32. De Ninno, G.; Mahieu, B.; Allaria, E.; Giannessi, L.; Spampinati, S. Chirped Seeded Free-Electron Lasers: Self-Standing Light Sources for Two-Color Pump-Probe Experiments. Phys. Rev. Lett. 2013, 110, 064801. [CrossRef] [PubMed]

33. Mahieu, B.; Allaria, E.; Castronovo, D.; Danailov, M.B.; Demidovich, A.; De Ninno, G.; Di Mitri, S.; Fawley, W.M.; Ferrari, E.; Frohlich, L.; et al. Two-colour generation in a chirped seeded Free-Electron Laser. Opt. Express 2013, 21, 022728. [CrossRef] [PubMed]

34. Principi, E.; Danailov, M.B.; Cucini, R.; D’Amico, F.; Pelizzo, M.G.; Mewes, L.-H.; Gessini, A.; Bencivenga, F.; Battistoni, A.; Giangrisostomi, E.; et al. Ultrafast dynamic of melting in Si monitored by tracking the $\mathrm{L}(2,3)$-edge shift. In preparation.

35. Gauthier, D.; Ribič, P.R.; De Ninno, G.; Allaria, E.; Cinquegrana, P.; Danailov, M.B.; Demidovich, A.; Ferrari, E.; Giannessi, L. Generation of Phase-Locked Pulses from a Seeded Free-Electron Laser. Phys. Rev. Lett. 2016, 116, 024801. [CrossRef] [PubMed]

36. Allaria, E.; Bencivenga, F.; Borghes, R.; Capotondi, F.; Castronovo, D.; Charalambous, P.; Cinquegrana, P.; Danailov, M.B.; De Ninno, G.; Demidovich, A.; et al. Two-colour pump-probe experiments with a twin-pulse-seed extreme ultraviolet free-electron laser. Nat. Commun. 2013, 4, 2476. [CrossRef] [PubMed]

37. Ferrari, E.; Spezzani, C.; Fortuna, F.; Delaunay, R.; Vidal, F.; Nikolov, I.; Cinquegrana, P.; Diviacco, B.; Gauthier, D.; Penco, G.; et al. Widely tunable two-colour seeded free-electron laser source for resonant-pump resonant-probe magnetic scattering. Nat. Commun. 2016, 7, 10343. [CrossRef] [PubMed]

38. Glover, T.E.; Fritz, D.M.; Cammarata, M.; Allison, T.K.; Sinisa Coh; Feldkamp, J.M.; Lemke, H.; Zhu, D.; Feng, Y.; Coffee, R.N.; et al. X-ray and optical wave mixing. Nature 2012, 488, 603-608. [CrossRef] [PubMed] 
39. Tamasaku, K.; Shigemasa, E.; Inubushi, Y.; Katayama, T.; Sawada, K.; Yumoto, H.; Ohashi, H.; Mimura, H.; Yabashi, M.; Yamauchi, K.; et al. X-ray two-photon absorption competing against single and sequential multiphoton processes. Nat. Photonics 2014, 8, 313-316. [CrossRef]

40. Shwartz, S.; Fuchs, M.; Hastings, J.B.; Inubushi, Y.; Ishikawa, T.; Katayama, T.; Reis, D.A.; Sato, T.; Tono, K.; Yabashi, M.; et al. X-ray Second Harmonic Generation. Phys. Rev. Lett. 2014, 112, 163901. [CrossRef] [PubMed]

41. Dhar, L.; Rogers, J.A.; Nelson, K.A. Time-Resolved Vibrational Spectroscopy in the Impulsive Limit. Chem. Rev. 1994, 94, 157-193. [CrossRef]

42. Hochstrasser, R.M. Two-dimensional spectroscopy at infrared and optical frequencies. Proc. Natl. Acad. Sci. USA 2007, 104, 14190-14196. [CrossRef] [PubMed]

43. Lewis, A.; Lieberman, K. Near-field optical imaging with a non-evanescently excited high-brightness light source of sub-wavelength dimensions. Nature 1991, 354, 214-216. [CrossRef]

44. Avisar, D.; Tannor, D.J. Complete Reconstruction of the Wave Function of a Reacting Molecule by Four-Wave Mixing Spectroscopy. Phys. Rev. Lett. 2011, 106, 170405. [CrossRef] [PubMed]

45. Bencivenga, F.; Cucini, R.; Capotondi, F.; Battistoni, A.; Mincigrucci, R.; Giangrisostomi, E.; Gessini, A.; Manfredda, M.; Nikolov, I.P.; Pedersoli, E.; et al. Four wave mixing experiments with extreme ultraviolet transient gratings. Nature 2015, 520, 205-208. [CrossRef] [PubMed]

46. Bencivenga, F.; Masciovecchio, C. FEL-based transient grating spectroscopy to investigate nanoscale dynamics. Nuc. Instrum. Methods Phys. Res. A 2009, 606, 785-789. [CrossRef]

47. Schirmacher, W.; Ruocco, G.; Scopigno, T. Acoustic Attenuation in Glasses and its Relation with the Boson Peak. Phys. Rev. Lett. 2007, 98, 025501. [CrossRef] [PubMed]

48. Bressler, Ch.; Milne, C.; Pham, V.-T.; ElNahhas, A.; van der Veen, R.M.; Gawelda, W.; Johnson, S.; Beaud, P.; Grolimund, D.; Kaiser, M.; et al. Femtosecond XANES Study of the Light-Induced Spin Crossover Dynamics in an Iron(II) Complex. Science 2009, 323, 489-492. [CrossRef] [PubMed]

49. Engel, G.S.; Calhoun, T.R.; Read, E.L.; Ahn, T.-K.; Mancal, T.; Cheng, Y.-C.; Blankenship, R.E.; Fleming, G.R. Evidence for wavelike energy transfer through quantum coherence in photosynthetic systems. Nature 2007, 446, 782-786. [CrossRef] [PubMed]

50. Marinelli, A.; Coffee, R.; Vetter, S.; Hering, P.; West, G.N.; Gilevich, S.; Lutman, A.A.; Li, S.; Maxwell, T.; Galayda, J.; et al. Optical Shaping of X-ray Free-Electron Lasers. Phys. Rev. Lett. 2016, 116, 254801. [CrossRef] [PubMed]

51. Roussel, E.; Ferrari, E.; Allaria, E.; Penco, G.; Di Mitri, S.; Veronese, M.; Danailov, M.; Gauthier, D.; Giannessi, L. Multicolor High-Gain Free-Electron Laser Driven by Seeded Microbunching Instability. Phys. Rev. Lett. 2015, 115, 214801. [CrossRef] [PubMed]

52. Grattoni, V.; Roussel, E.; Allaria, E.; Di Mitri, S.; Giannessi, L.; Ferrari, E.; Sigalotti, P.; Penco, G.; Veronese, M.; Badano, L.; et al. Control of the Seeded Fel Pulse Duration Using Laser Heater Pulse Shaping. Presented at the IPAC'17, Copenhagen, Denmark, 14-19 May 2017. Paper Number WEPAB034.

53. Giannessi, L.; Musumeci, P.; Spampinati, S. Nonlinear pulse evolution in seeded free-electron laser amplifiers and in free-electron laser cascades. J. Appl. Phys. 2005, 98, 043110. [CrossRef]

54. Watanabe, T.; Wang, X.J.; Murphy, J.B.; Rose, J.; Shen, Y.; Tsang, T.; Giannessi, L.; Musumeci, P.; Reiche, S. Experimental Characterization of Superradiance in a Single-Pass High-Gain Laser-Seeded Free-Electron Laser Amplifier. Phys. Rev. Lett. 2007, 98, 034802. [CrossRef] [PubMed]

55. Giannessi, L.; Artioli, M.; Bellaveglia, M.; Briquez, F.; Chiadroni, E.; Cianchi, A.; Couprie, M.E.; Dattoli, G.; Di Palma, E.; Di Pirro, G.; et al. High-order-harmonic generation and superradiance in a seeded free-electron laser. Phys. Rev. Lett. 2012, 108, 164801. [CrossRef] [PubMed]

56. Stupakov, G. Using the Beam-Echo Effect for Generation of Short-Wavelength Radiation. Phys. Rev. Lett. 2009, 102, 74801. [CrossRef] [PubMed]

57. Xiang, D.; Stupakov, G. Echo-enabled harmonic generation free electron laser. Phys. Rev. Spec. Top. Accel. Beams 2009, 12, 30702. [CrossRef]

58. Hemsing, E.; Dunning, M.; Garcia, B.; Hast, C.; Raubenheimer, T.; Stupakov, G.; Xiang, D.; et al. Echo-enabled harmonics up to the 75th order from precisely tailored electron beams. Nat. Photonics 2016, 10, 512-515. [CrossRef]

59. Rebernik Ribic, P.; Roussel, E.; Penn, G.; De Ninno, G.; Giannessi, L.; Penco, G.; Allaria, E. Echo-Enabled Harmonic Generation Studies for the FERMI Free Electron Laser. Photonics 2017, 4, 19. [CrossRef] 
60. Penco, G.; Allaria, E.; Bassanese, S.; Cinquegrana, P.; Cleva, S.; Danailov, M.B.; Demidovich, A.A.; Ferianis, M.; Gaio, G.; Gauthier, D.; et al. Two-Bunch Operation at the FERMI FEL Facility. Presented at the IPAC'17, Copenhagen, Denmark, 14-19 May 2017. Paper Number WEPAB037.

61. Bennett, K.; Zhang, Y.; Kowalewski, M.; Hua, W.; Mukamel, S. Multidimensional resonant nonlinear spectroscopy with coherent broadband X-ray pulses. Phys. Scr. 2016, 014002. [CrossRef] 